\title{
Solution of One-dimensional Partial Differential Equation with Higher-Order Derivative by Double Laplace Transform Method
}

\author{
Anongo D.O. and Awari Y.S. \\ Department of Mathematical Sciences, State University, Jalingo \\ Email: awari@tsuniversity.edu.ng
}

Cite this article:

Anongo D.O., Awari Y.S. (2021), Solution of Onedimensional Partial Differential Equation with Higher-Order Derivative by Double Laplace Transform Method. African Journal of Mathematics and Statistics Studies 4(3), 1-11. DOI: 10.52589/AJMSS1OHGJPNR.

\section{Manuscript History}

Received: 13 July 2021

Accepted: 7 Aug 2021

Published: 21 Aug 2021

Copyright $\odot 2020$ The Author(s). This is an Open Access article distributed under the terms of Creative Commons AttributionNonCommercial-NoDerivatives 4.0 International (CC BY-NC-ND 4.0 ), which permits anyone to share, use, reproduce and redistribute in any medium, provided the original author and source are credited.
ABSTRACT: Many problems in natural and engineering sciences such as heat transfer, elasticity, quantum mechanics, water flow, and others are modelled mathematically by partial differential equations. Some of these problems may be linear, nonlinear, homogeneous, non-homogeneous, and order greater or equal one. Finding the theoretical solution to these problems with less cumbersome techniques is an active area of research in the aforementioned field. In this research paper, we have developed a new application of the double Laplace transform method to solve homogeneous and non-homogeneous linear partial differential equations ( $p d e s$ ) with higher-order derivatives (i.e order $n$ where $n \geq 2$ ) in science and engineering. We discussed a brief theory of double Laplace transforms that helped in its application. The main advantage of our method is the reduction of computational effort in finding solution to pdes. Another major benefit of our method is solving problems in the form of (21) directly by transforming to an algebraic equation where the inverse double Laplace transform is implemented for analytical solution, unlike other integral transform methods that would first transform to a system of ODEs before they are solved, is it also very effective in solving linear high-order partial differential equations and yield fast convergence. We present a well-simplified solution for easier comprehension by upcoming researchers.

KEYWORDS: Double-Laplace Tranform, Partial Differential Equations, Boundary Value Problems, Exponential Order, Fluid Dynamics. 


\section{INTRODUCTION}

Many problems in natural and engineering sciences are modelled mathematically by differential equations [13], [10] and [16]. In physics, for instance, the heat flow and the wave propagation phenomena are well defined by partial differential equations [20]. The dispersion of a chemically reactive material is characterized by partial differential equations [7]. In addition, most physical phenomena of fluid dynamics, quantum mechanics, electricity, plasma physics, propagation of shallow-water waves, chemical reaction-diffusion, and many other models are governed by partial differential equations [14] and [8]. Some of these problems may be linear, nonlinear, homogeneous, non-homogeneous, and order greater or equal one [2]. Finding the exact solution to these problems with a less cumbersome technique is active research in the aforementioned field [7]. Laplace and Fourier's transforms were found very effective for the solution of linear ordinary differential equations [9]. In the case of linear partial differential equations such as wave, heat, or Laplace equation similar consideration hold, however with much difficulty as the ODEs that are evolving are often complicated and the inverse Laplace transform does not exist for them [9], this method failed for many linear PDEs especially of variable coefficient [9]. The method of characteristics works well with generally first order and hyperbolic PDEs [17] and the method of separation of variables works generally for most second-order linear PDEs [19] and [1]. The third-order linear dispersive wave equation and fourth-order linear Euler Bernoulli equation govern long water waves in relatively shallow water for very small amplitude and the deflection of an elastic beam under the action of a load [11] respectively are higher-order PDEs. The solution obtained by Homotopy Perturbation Method for linear Partial differential equations in some cases is divergent [12]. The authors [3] used the Sumudu transform to solve the linear telegraphic equation, the transform resulted in a complicated ODE that its solution form utterly another problem. Moreover, [4] use the numerical discontinuous Galerkin finite element method to solve linear time-dependent partial differential equations with higher-order derivatives but it is important to investigate the analytical solution in other to understand phenomena between them [7]. Debnath (2016) applied the double Laplace transform to solve the second-order wave and the heat equation, consequence of his idea; the main aim of this research is to develop a new application of double Laplace transform to solve homogeneous and non-homogeneous higherorder linear partial differential equations in science and engineering.

\section{BRIEF THEORY OF DOUBLE LAPLACE TRANSFORM}

Let $f(x, t)$ be a function of two variables $x$ and $t$ defined in the positive quadrant of the $x t$ plane. The double Laplace transform of the function $f(x, t)$ as given by [18] is defined by

$$
L_{x} L_{t}\{f(x, t)\}=F(p, s)=\int_{0}^{\infty} \quad e^{-p x} \int_{0}^{\infty} e^{-s t} f(x, t) d t d x
$$

whenever that integral exists, here $p$ and $s$ are complex numbers and $x, t>0$

The inverse double Laplace transform, $L_{x}^{-1} L_{t}^{-1}\{F(p, s)\}=f(x, t)$ is defined by [5] as the complex double integral formula 
$L_{x}^{-1} L_{t}^{-1}\{F(p, s)\}=f(x, t)=\frac{1}{2 \pi i} \int_{d-i \infty}^{c+i \infty} \quad e^{p x} d p \frac{1}{2 \pi i} \int_{d-i \infty}^{c+i \infty} \quad e^{s t} F(p, s) d s$

where $F(p, s)$ must be an analytic function for all $p$ and $s$ in the region defined by the inequalities $\operatorname{Re} p \geq c$ and $\operatorname{Re} s \geq d$ where c and $\mathrm{d}$ are real constants to be chosen suitably, where we follow [15] to define the Laplace transform of a function $f(t), t>0$ as $L\{f(t)\}=F(s)=\int_{0}^{\infty} e^{-s t} f(t) d t, \operatorname{Re}(s)>0$

similarly

The inverse Laplace transform, $L_{t}^{-1}\{F(s)\}=f(t)$ is defined by [5] as the complex double integral formula

$L_{t}^{-1}\{F(s)\}=f(t)=\frac{1}{2 \pi i} \int_{c-i \infty}^{c+i \infty} \quad e^{s t} F(s) d s, c \geq 0$

(4)

\section{Double Laplace Transform of Partial Derivative}

Consider the partial differential equation of the form [8],

$\sum_{n=0}^{N} \quad a_{n} \frac{\partial^{n} u(x, t)}{\partial t^{n}}=\sum_{m=1}^{M} \quad b_{m} \frac{\partial^{m} u(x, t)}{\partial x^{m}}+f(x, t),(x, t) \in R_{+}^{2}$

Where $a_{n}, 0 \leq n \leq N ; 1 \leq m \leq M$ are given coefficients, $N, M$ are positive integers, $a, b$ are integers and $f(x, t)$, is the source term and the following initial and boundary conditions respectively

$\frac{\partial^{n} u(x, 0)}{\partial t^{n}}=g_{n}(x), n=0,1, \ldots \ldots \ldots, N-1, x \in R_{+}$

(6)

and

$\frac{\partial^{m} u(0, t)}{\partial t^{m}}=f_{m}(t), n=0,1, \ldots \ldots \ldots, M-1, t \in R_{+}$

(7)

Further, we assume that the function $f, g_{n}, n=0,1, \ldots \ldots \ldots, N-1$ and $f_{m}, m=0,1, \ldots, M-1$ are such that problems (1.1), (1.2), and (1.3) have solution(s).

$L_{x} L_{t}\left\{\frac{\partial u(x, t)}{\partial t}\right\}=s U(p, s)-L_{x}\{u(x, 0)\}$

(8)

$$
\begin{aligned}
& L_{x} L_{t}\left\{\frac{\partial^{2} u(x, t)}{\partial t^{2}}\right\}=s^{2} U(p, s)-s L_{x}\{u(x, 0)\}-L_{x}\left\{\frac{\partial u(x, 0)}{\partial t}\right\} \\
& L_{x} L_{t}\left\{\frac{\partial^{n} u(x, t)}{\partial t^{n}}\right\}=s^{n} U(p, s)-\sum_{k=0}^{n-1} \quad s^{n-1-k} L_{x}\left\{\frac{\partial^{k} u(x, 0)}{\partial t^{k}}\right\}
\end{aligned}
$$


And

$L_{x} L_{t}\left\{\frac{\partial u(x, t)}{\partial x}\right\}=p U(p, s)-L_{t}\{u(0, t)\}$

$L_{x} L_{t}\left\{\frac{\partial^{2} u(x, t)}{\partial x^{2}}\right\}=p^{2} U(p, s)-p L_{t}\{u(0, t)\}-L_{t}\left\{\frac{\partial u(0, t)}{\partial x}\right\}$

$L_{x} L_{t}\left\{\frac{\partial^{3} u(x, t)}{\partial x^{3}}\right\}=s^{3} U(p, s)-s^{2} L_{t}\{u(0, t)\}-s L_{t}\left\{\frac{\partial u(0, t)}{\partial x}\right\}-L_{t}\left\{\frac{\partial^{2} u(0, t)}{\partial x^{2}}\right\}$

$L_{x} L_{t}\left\{\frac{\partial^{4} u(x, t)}{\partial x^{4}}\right\}=s^{4} U(p, s)-s^{3} L_{t}\{u(0, t)\}-s^{2} L_{t}\left\{\frac{\partial u(0, t)}{\partial x}\right\}-s L_{t}\left\{\frac{\partial^{2} u(0, t)}{\partial x^{2}}\right\}-$

$L_{t}\left\{\frac{\partial^{3} u(0, t)}{\partial x^{3}}\right\}(13)$

$L_{x} L_{t}\left\{\frac{\partial^{m} u(x, t)}{\partial x^{m}}\right\}=p^{m} U(p, s)-\sum_{j=0}^{m-1} \quad p^{m-1-j} L_{t}\left\{\frac{\partial^{j} u(x, 0)}{\partial x^{j}}\right\}$

and

$L_{x} L_{t}\left\{\frac{\partial^{2} u(x, t)}{\partial x \partial t}\right\}=p s U(p, s)-U(0, s)-U(p, 0)-U(0,0)$

\section{Exponential Order}

Debnath, (2016) stated that a function $f(x, t)$ is said to be of exponential order $a(>0)$ and $b(>0)$ on $0 \leq x<\infty$, if there exists a positive constant $k$ such that for all $x>X$ and $t>T$

$|f(x, t)| \leq K e^{a x+b t}$

And we write

$f(x, t)=0\left(e^{a x+b t}\right)$ as $x \rightarrow \infty, t \rightarrow \infty$

$(17)$

$e^{-a x-b t}|f(x, t)|=K e^{-(\alpha-a) x} e^{-(\beta-b) t}=0, \alpha>a, \beta>b$

Such a function $f(x, t)$ is simply called an exponential order as $x \rightarrow \infty, t \rightarrow \infty$ and clearly, it does not grow faster than $K e^{a x+b t}$ as $x \rightarrow \infty, t \rightarrow \infty$

\section{Existence Condition for Double Laplace Transform}

Debnath (2016) stated that if a function $f(x, t)$ is a continuous function in every finite interval $(0, X)$ and $(0, T)$ and of exponential order $e^{a x+b t}$, then the double Laplace transform of $f(x, t)$ exist for all $p$ and $s$ provided $\operatorname{Re} p>a$ and $\operatorname{Re} s>b$. 
Proof

we have that,

$|F(p, s)|=\left|\iint_{0}^{\infty} e^{-p x-s t} f(x, t) d x d t\right|$

$$
\begin{aligned}
& \leq K \int_{0}^{\infty} e^{-x(p-a)} d x \int_{0}^{\infty} e^{-t(s-b)} d t \\
= & \frac{K}{(p-a)(s-b)} \text { for } \operatorname{Re} p>a, \operatorname{Re} s>b
\end{aligned}
$$

It follows that

$$
|F(p, s)|=0 \operatorname{or} F(p, s)=0
$$

This result can be regarded as the limiting property of the double Laplace transform. Clearly, $F(p, s)=p s$ or $p^{2}+s^{2}$ is not the double Laplace transform of any function $f(x, t)$ because $F(p, s)$ does not tend to zero as $p \rightarrow \infty$ and $s \rightarrow \infty$.

On the other hand, $f(x, t)=e^{a x^{2}+b t^{2}}, a>0, b>0$ cannot have a double Laplace transform even though it is continuous but is not of the exponential order because

$$
e^{a x^{2}+b t^{2}-p x-s t}=\infty
$$

\section{Table of Double Laplace transform for some function of two variables}

Using [6], the following properties hold on $f(x, t)$

$$
\begin{array}{lc}
L_{x} L_{t}\{f(x, t)\} & F(p, s) \\
1 & \frac{1}{p q} \\
e^{(a x+b t)} & \frac{1}{(p-a)(s-b)} \\
e^{i(a x+b t)} & \frac{(p s-a b)+i(a s+b p)}{\left(p^{2}+a^{2}\right)\left(s^{2}+b^{2}\right)} \\
\cos (a x+b t) & \frac{p s-a b}{\left(p^{2}+a^{2}\right)\left(s^{2}+b^{2}\right)} \\
\sin (a x+b t) & \frac{a s-b p}{\left(p^{2}+a^{2}\right)\left(s^{2}+b^{2}\right)} \mathrm{k} \\
\cosh \cosh (a x+b t) & \frac{1}{2}\left[\frac{1}{(p-a)(s-b)}+\frac{1}{(p+a)(s+b)}\right] \\
\sinh (a x+b t) & \left.\frac{1}{(p-a)(s-b)}-\frac{1}{(p+a)(s+b)}\right] \\
e^{-a x-b t} f(x, t) & F(p+a, s+b) \\
(x t)^{n}
\end{array}
$$




$$
\begin{array}{lc}
g(x) h(t) & G(P) H(s) \\
f(a x) g(b t) & \frac{1}{a b} F\left(\frac{p}{a}\right) G\left(\frac{s}{b}\right) a>0, b>0 \\
\frac{\partial f(x, t)}{\partial x} & p F(p, s)-F(0, s) \\
\frac{\partial^{2} f(x, t)}{\partial^{2} x} & p^{2} F(p, s)-p F(p, s)-F(0, s)-\frac{\partial F(0, s)}{\partial x} \\
\frac{\partial^{2} f(x, t)}{\partial x \partial t} & p s F(p, s)-p F(p, 0)-s F(0, s)-F(0,0)
\end{array}
$$

\section{APPLICATION TO HIGHER ORDER PARTIAL DIFFERENTIAL EQUATION}

In this chapter, we present three (3) problems to illustrates the efficiency, applicability, and accuracy of the double Laplace transform method to solve higher-order homogenous and nonhomogenous boundary value problems which include second-order linear Klein-Gordon equation, linear third-order dispersive equation, and the fourth-order Euler-Bernoulli Equation

Example 3.1 Solve the homogeneous Klein-Gordon equation

$\frac{\partial^{2} u(x, t)}{\partial t^{2}}-u(x, t)=\frac{\partial^{2} u(x, t)}{\partial x^{2}},(x, t) \epsilon R_{+}^{2}$

with initial and boundary conditions

$u(x, 0)=1+\sin x=g_{0}(x), \frac{\partial u(x, 0)}{\partial t}=0=g_{1}(x), x \in R_{+}$

$u(0, t)=\cosh t=f_{0}(t), \frac{\partial u(0, t)}{\partial x}=1=f_{1}(t), t \in R_{+}$

The Klein-Gordon equation plays an important role in the study of solutions in condensed matter physics, quantum mechanics, and relativistic physics [7].

Solution Taking the double Laplace transform on both sides of (21), we have

$L_{x} L_{t}\left[\frac{\partial^{2} u(x, t)}{\partial t^{2}}\right]-L_{x} L_{t}[u(x, t)]=L_{x} L_{t}\left[\frac{\partial^{2} u(x, t)}{\partial x^{2}}\right]$

$s^{2} U(p, s)-s L_{x}\{u(x, 0)\}-L_{x}\left\{\frac{\partial u(x, 0)}{\partial t}\right\}-U(p, s)=p^{2} U(p, s)-p L_{t}\{u(0, t)\}-$ $L_{t}\left\{\frac{\partial u(0, t)}{\partial x}\right\}(25)$

Taking the single Laplace transform of the initial and boundary conditions, we have $L_{x}\{u(x, 0)\}=G_{0}(p)=\frac{1}{p}+\frac{1}{p^{2}+1}, L_{x}\left\{\frac{\partial u(x, 0)}{\partial t}\right\}=G_{1}(p)=0$ 
$L_{t}\{u(0, t)\}=F_{0}(s)=\frac{s}{s^{2}-1}, L_{t}\left\{\frac{\partial u(0, t)}{\partial x}\right\}=F_{1}(s)=\frac{1}{s}$

Substituting (26 \& 27) into (25), we have

$u(p, s)=\frac{s G_{o}(p)+G_{1}(p)-p F_{0}(s)-F_{1}(s)}{\left(s^{2}-1-p^{2}\right)}$

Simplifying and applying the inverse double Laplace transform (2), we obtained the solution as

$u(x, t)=L_{x}^{-1} L_{t}^{-1}\left[\frac{1}{\left(s^{2}-1-p^{2}\right)}\left[s\left(\frac{1}{p}+\frac{1}{p^{2}+1}\right)-p \frac{s}{s^{2}-1}-\frac{1}{s}\right]\right]$

Simplifying, we obtain

$u(x, t)=L_{x}^{-1} L_{t}^{-1}\left[\frac{1}{\left(s^{2}-1-p^{2}\right)}\left[\frac{\left(s^{2}-1-p^{2}\right)}{s\left(p^{2}+1\right)}+\frac{s\left(s^{2}-1-p^{2}\right)}{p\left(s^{2}-1\right)}\right]\right]$

$u(x, t)=L_{x}^{-1} L_{t}^{-1}\left[\frac{1}{s\left(p^{2}+1\right)}+\frac{s}{p\left(s^{2}-1\right)}\right]$

$u(x, t)=\sin x+\cosh t$

Example 3.2 Solve the linear third-order dispersive non-homogeneous equation. It governs long water waves in relatively shallow water for very small amplitude [11].

$\frac{\partial u(x, t)}{\partial t}=\frac{\partial^{3} u(x, t)}{\partial x^{3}}+2 e^{t-x},(x, t) \in R_{+}^{2}$

with initial and boundary conditions

$u(x, 0)=1+e^{-x}=g_{0}(x), x \in R_{+}$

$u(0, t)=1+e^{t}=f_{0}(t), \frac{\partial u(0, t)}{\partial x}=-e^{t}=f_{1}(t), \frac{\partial^{2} u(0, t)}{\partial x^{2}}=e^{t}=f_{2}(t), t \in R_{+}$

Solution Taking the double Laplace transform on both sides of (33), we have

$L_{x} L_{t}\left[\frac{\partial u(x, t)}{\partial t}\right]=L_{x} L_{t}\left[\frac{\partial^{3} u(x, t)}{\partial x^{3}}\right]+L_{x} L_{t}\left[2 e^{t-x}\right]$

$s U(p, s)-L_{x}\{u(x, 0)\}=s^{3} U(p, s)-s^{2} L_{t}\{u(0, t)\}-s L_{t}\left\{\frac{\partial u(0, t)}{\partial x}\right\}-L_{t}\left\{\frac{\partial^{2} u(0, t)}{\partial x^{2}}\right\}+$ $F(p, s)(37)$

Where $F(p, s)=\frac{2}{(s-1)(p+1)}$ 
Taking the single Laplace transform of the initial and boundary conditions, we have

$L_{x}\{u(x, 0)\}=G_{0}(p)=\frac{1}{p}+\frac{1}{p+1}$

$L_{t}\{u(0, t)\}=F_{0}(s)=\frac{1}{s}+\frac{1}{s+1}, L_{t}\left\{\frac{\partial u(0, t)}{\partial x}\right\}=F_{1}(s)=\frac{-1}{s-2}, L_{t}\left\{\frac{\partial^{2} u(0, t)}{\partial x^{2}}\right\}=F_{2}(s)=\frac{1}{s-2}$,

Substituting (38), (39) \& (40) into (37), we have

$u(p, s)=\frac{G_{0}(p)-p^{2} F_{0}(s)-p F_{1}(s)-F_{2}(s)+F(p, s)}{s-p^{3}}$

Simplifying and taking the inverse double Laplace transform (2), we have

$$
u(x, t)=L_{x}^{-1} L_{t}^{-1}\left[\frac{1}{\left(s-p^{3}\right)}\left[\frac{1}{p}+\frac{1}{p+1}-p^{2}\left(\frac{1}{s}+\frac{1}{s-1}\right)+p \frac{1}{s-1}-\frac{1}{s-1}+\frac{2}{(s-1)(p+1)}\right]\right]
$$

Simplifying, we obtain the solution

$$
\begin{aligned}
& u(x, t)=L_{x}^{-1} L_{t}^{-1}\left[\frac{1}{\left(s-p^{3}\right)}\left(\frac{s-p^{3}}{p s}+\frac{s-p^{3}}{(p+1)(s-1)}\right)\right] \\
& u(x, t)=L_{x}^{-1} L_{t}^{-1}\left[\frac{1}{p s}+\frac{1}{(p+1)(s-1)}\right] \\
& u(x, t)=1+e^{t-x}
\end{aligned}
$$

\section{Example 3.3 The Euler-Bernoulli Equation}

It governs the deflection of an elastic beam under the action of a load [11].

$-\frac{\partial^{2} u(x, t)}{\partial t^{2}}=\frac{\partial^{4} u(x, t)}{\partial x^{4}}-x t-t^{2},(x, t) \epsilon R_{+}^{2}$

with initial and boundary conditions

$$
\begin{aligned}
& u(x, 0)=0=g_{0}(x), \frac{\partial u(x, 0)}{\partial t}=\frac{x^{5}}{5 !}=g_{1}(x), x \in R_{+} \\
& u(0, t)=\frac{x^{4}}{12}=f_{0}(t), \frac{\partial u(0, t)}{\partial x}=0=f_{1}(t), \frac{\partial^{2} u(0, t)}{\partial x^{2}}=0=f_{2}(t), \frac{\partial^{3} u(0, t)}{\partial x^{3}}=0=f_{3}(t), t \in R_{+}
\end{aligned}
$$

Solution Taking the double Laplace transform on both sides of (46), we have

$$
\begin{aligned}
& L_{x} L_{t}\left[-\frac{\partial^{2} u(x, t)}{\partial t^{2}}\right]=L_{x} L_{t}\left[\frac{\partial^{4} u(x, t)}{\partial x^{4}}\right]-L_{x} L_{t}\left[x t+t^{2}\right] \\
& s^{2} U(p, s)+s L_{x}\{u(x, 0)\}+L_{x}\left\{\frac{\partial u(x, 0)}{\partial t}\right\}=s^{4} U(p, s)-s^{3} L_{t}\{u(0, t)\}-s^{2} L_{t}\left\{\frac{\partial u(0, t)}{\partial x}\right\}- \\
& s L_{t}\left\{\frac{\partial^{2} u(0, t)}{\partial x^{2}}\right\}-L_{t}\left\{\frac{\partial^{3} u(0, t)}{\partial x^{3}}\right\}-F(p, s)
\end{aligned}
$$


Where $F(p, s)=-\frac{1}{p^{2} s^{2}}-\frac{2}{p s^{3}}$

Taking the single Laplace transform of the initial and boundary conditions, we have

$$
\begin{aligned}
& L_{x}\{u(x, 0)\}=G_{0}(p)=0, L_{x}\left\{\frac{\partial u(x, 0)}{\partial t}\right\}=G_{1}(p)=\frac{1}{p^{6}} \\
& L_{t}\{u(0, t)\}=F_{0}(s)=\frac{2}{s^{5}}, L_{t}\left\{\frac{\partial u(0, t)}{\partial x}\right\}=F_{1}(s)=0, L_{t}\left\{\frac{\partial^{2} u(0, t)}{\partial x^{2}}\right\}=F_{2}(s)=0 \\
& L_{t}\left\{\frac{\partial^{3} u(0, t)}{\partial x^{3}}\right\}=F_{3}(s)=0
\end{aligned}
$$

Substituting (51), (52), (53) \& (54), we have

$u(p, s)=\frac{s G_{0}(p)+G_{1}(p)+\left\{p^{3} F_{0}(s)+p^{2} F_{1}(s)+p F_{2}(s)+F_{3}(s)\right\}-F(p, s)}{s^{2}+p^{4}}$

Simplifying and taking the inverse double Laplace transform (2), we have

$$
u(x, t)=L_{x}^{-1} L_{t}^{-1}\left[\frac{1}{\left(p^{4}+s^{2}\right)}\left[\frac{1}{p^{6}}+p^{3} \frac{2}{s^{5}}+\frac{1}{p^{2} s^{2}}+\frac{2}{p s^{3}}\right]\right]
$$

Simplifying, we obtain the solution

$$
u(x, t)=L_{x}^{-1} L_{t}^{-1}\left[\frac{1}{\left(p^{4}+s^{2}\right)}\left(\frac{p^{4}+s^{2}}{p^{6} s^{2}}+\frac{2\left(p^{4}+s^{2}\right)}{p s^{5}}\right)\right]
$$

$$
\begin{aligned}
& u(x, t)=L_{x}^{-1} L_{t}^{-1}\left[\frac{1}{p^{6} s^{2}}+\frac{2}{p s^{5}}\right] \\
& u(x, t)=\frac{t x^{5}}{5 !}+\frac{t^{4}}{12}
\end{aligned}
$$

\section{CONCLUSION}

We have applied double Laplace transform to obtain exact solutions for linear homogeneous and non-homogeneous higher order partial differential equations. All of the examples considered show that the double Laplace transform method is capable of reducing the volume of computational work, solves problems directly by transforming to an algebraic equation where the inverse double Laplace transform is implemented for exact solution, unlike other integral transform methods that would first transform to a system of ODEs before they are solved, very effective in solving linear high order partial differential equations and yield fast convergence.

\section{Competing Interests}

The authors declare no competing interests regarding the publication of this paper 


\section{REFERENCE}

[1] Birkhäuser B. (2007) of Separation of Variables. Linear Partial Differential Equations for Scientists and Engineerhttps://doi.org/10.1007/978-0-8176-4560-1_7

[2] Borthwick, D., (2016). Introduction to Partial Differential Equation, the springer international publishing. Doi10.1007/978-3-319-48936-0

[3] Briones, R. P. (2020). On the Solution of Partial Differential Equations Using the Sumudu Transform. International Journal of Mathematical Analysis, 14(8), 389-395.

[4] Cheng, Y., \& Shu, C. W. (2008). A discontinuous Galerkin finite element method for time-dependent partial differential equations with higher-order derivatives. Mathematics of computation, 77(262), 699-730.

[5] Debnath, L. (2016). The double Laplace transforms and their properties with applications to Functional, Integral, and Partial Differential Equations. Int. J. Appl. Comput. Math, 2, 223-241. DOI 10.1007/s40819-015-0057-3

[6] Debnath, L. and Batta, D. (2015). Integral Transforms and Their Applications, $3^{\text {rd }}$ edition. CRC Press, Chapman \& Hall, Boca Raton

[7] Deniz, S., \&Bildik, N. (2014). Comparison of Adomian decomposition method and Taylor matrix method in solving different kinds of partial differential equations. International Journal of Modeling and Optimization, 4(4), 292.

[8] Dhunde, R. R., \&Waghmare, G. L. (2017). Double Laplace transform method in mathematical physics. International journal of theoretical and mathematical physics, 7(1), 14-20.

[9] Duffy, D.G. (2004). Transform Methods for Solving Partial Differential Equations (2nd ed.). Chapman and Hall/CRC. https://doi.org/10.1201/9781420035148

[10] Khan, A., Khan, T. S., Syam, M. I., \& Khan, H. (2019). Analytical solutions of the time-fractional wave equation by double Laplace transform method. The European Physical Journal Plus, 134(4), 163.

[11] Lesnic, D. (2006). The Decomposition method for Linear, one-dimensional, timedependent partial differential equations. International Journal of Mathematics and Mathematical Sciences, Volume 2006, 1-29.

[12] Liang, S., \& Jeffrey, D. J. (2009). Comparison of homotopy analysis method and homotopy perturbation method through an evolution equation. Communications in Nonlinear Science and Numerical Simulation, 14(12), 4057-4064.

[13] Logan, J. D. (2015). Applied Partial Differential Equations ( $3^{\text {rd }}$ ed.). Springer international publishing. https://doi10.1007/978-3-319-12493-3

[14] Ming, C. Y. (2017). Solution of differential equation with applications to engineering problems. Dynamical Systems, Analytical and Computational Techniques, 234-264. http.//dx.doi.org/10.5772/67539

[15] Morales-Delgado, V. F., Taneco-Hernández, M. A., \& Gómez-Aguilar, J. F. (2017). On the solutions of fractional order of evolution equations. The European Physical Journal Plus, 132(1), 1-14.

[16] Opanuga, A. A., Owoloko, E. A., \&Okagbue, H. I. (2017). Comparison homotopy perturbation and Adomian decomposition techniques for parabolic equations. Proceedings of the World Congress on Engineering 
[17] Sarra, S. A. (2003). The method of characteristics with applications to conservation laws. Journal of Online Mathematics and its Applications, 3, 1-16.

[18] Sneddon I. N. (1974). The use of integral transform, Tata McGraw Hill Edition

[19] Subramanian, S. R. (2019). Solution of partial differential equations: separation of variables. Department of Chemical and Biomolecular Engineers Research Gate,1-8, https://www.researchgate.net/publication/332593615

[20] Yang, X. J. (2017). A new integral transform operator for solving the heat-diffusion problem. Applied Mathematics Letters, 64, 193-197 